\title{
A case of life-threatening post-operative diffuse alveolar hemorrhage in patient with recent chemotherapy -A case report-
}

\author{
Mi-Young Kwon ${ }^{1}$, Yoon Kyung Lee ${ }^{2}$, In-Cheol Choi ${ }^{2}$, Eun Ho Lee ${ }^{2}$, Nam-Yun Kim , and Young-Jae Chang \\ Department of Anesthesiology and Pain Medicine, ${ }^{1}$ National Medical Center, ${ }^{2}$ Asan Medical Center, College of Medicine, University \\ of Ulsan, Seoul, Korea
}

\begin{abstract}
A 53-year-old woman who had undergone total gastrectomy and received adjuvant chemotherapy two months ago underwent adhesiolysis of the small bowel. She presented with sudden desaturation and dyspnea of unknown etiology at postanesthetic care unit. Following ET intubation, the endotracheal tube suction revealed massive hemoptysis. Bilateral lung infiltrated on her chest radiograph and bronchofibroscopic examination disclosed a diffuse hemorrhage on both lung fields without bleeding focus. These findings were consistent with diffuse alveolar hemorrhage (DAH) syndrome. As per our knowledge and search, this is the first reported case of DAH that occurred during the recovery period immediately after general anesthesia. DAH is known to have a high mortality rate and an early detection followed by adequate treatment is essential. (Korean J Anesthesiol 2011; 60: 221-225)
\end{abstract}

Key Words: Dyspnea, Hemoptysis, Hemorrhage.

Diffuse alveolar hemorrhage (DAH) syndrome is caused by disruption of the alveolar-capillary basement membrane which results in bleeding into the alveolar spaces. There is a sudden flow of blood into multiple sites of alveoli and may culminate into an acute, life-threatening event. A variety of diseases and drugs have been associated with this syndrome [1]. An exposure to infectious or toxic agents, systemic vasculitis (e.g., Behcet's syndrome, Henoch-Schoenlein purpura and Wegener's granulomatosis), connective tissue diseases (e.g., Goodpasture's syndrome and systemic lupus erythematosis) and drugs (e.g., amiodarone and penicillamine) are well-known etiologic factors. DAH is relatively difficult to diagnose and is considered potentially fatal. In most cases, the etiology is not always clear, even in the event of severe hemorrhage. However, In spite of this fact, early intervention of DAH justifies a successful recovery. Thus, all the clinicians should be made familiar to DAH in order to enable them to arrive at an early diagnosis even during the initial examination of the patient.

We report an unexpected and potentially fatal case of DAH that occurred during immediate postoperative period at the post-anesthetic care unit, in which a case of massive alveolar hemorrhage was managed immediately and successfully. The patient underwent adhesiolysis for an hour due to mechanical obstruction after the total gastrectomy.

Received: June 12, 2010. Revised: 1st, August 2, 2010; 2nd, August 17, 2010. Accepted: October 15, 2010.

Corresponding author: Yoon Kyung Lee, M.D., Ph.D., Department of Anesthesiology and Pain Medicine, Asan Medical Center, College of Medicine, University of Ulsan, 388-1, Pungnap-dong, Songpa-gu, Seoul 138-736, Korea. Tel: 82-2-3010-3868, Fax: 82-2-470-1363, E-mail: liahi@dreamwiz.com (c) This is an open-access article distributed under the terms of the Creative Commons Attribution Non-Commercial License (http:// creativecommons.org/licenses/by-nc/3.0/), which permits unrestricted non-commercial use, distribution, and reproduction in any medium, provided the original work is properly cited. 


\section{Case Report}

A 53-year-old female patient, $161 \mathrm{~cm}$ and $46 \mathrm{~kg}$ was diagnosed with mechanical ileus and admitted for adhesiolysis. She underwent total gastrectomy for cancer of stomach 2 months ago and had received adjuvant chemotherapy three weeks ago; 2nd time with S-1 (TS-1 ${ }^{\circledR}$, Jeil Pharmaceutical Co., Ltd, Seoul, Korea) $60 \mathrm{mg}$ bid, for 14 days plus cisplatin (Cispuran ${ }^{\circledR}$, Dong-A Pharmaceutical Co., Ltd, Seoul, Korea) $60 \mathrm{mg} / \mathrm{m}^{2}$ on day 1. There was no previous history of cardiopulmonary disease, allergies, tuberculosis exposure, or smoking.

On her visit to emergency room, vital signs of the patient were as follows: body temperature, $37.2^{\circ} \mathrm{C}$; heart rate, 106 beats/minute with a regular rhythm; $\mathrm{SpO}_{2}, 100 \%$ (on room air); and blood pressure, 116/86 mmHg. Physical examination revealed distended and diminished bowel sounds. Laboratory findings on ER were: white blood cell (WBC) count, $3.3 \times 103$ / $\mathrm{mm}^{3}$; hemoglobin (Hb), $12.8 \mathrm{~g} / \mathrm{dl}$; platelet count, $184 \times 103 /$ $\mathrm{mm}^{3}$; C-reactive proteins (CRP), $0.56 \mathrm{mg} / \mathrm{dl}$; PT (INR), 1.05 (normal, 0.8-1.3); and activated PTT, $20.9 \mathrm{sec}$ (normal, 25.035.0). A preoperative chest X-ray, pulmonary function test and electrocardiogram were within the normal range. She was premedicated by oral midazolam (3.75 mg).

After the patient was taken to the operating room, she was on ECG standard leads II, noninvasive monitors for blood pressure, heart rate, arterial oxygen saturation, capnogram, and bispectral index. The patient underwent 3 minutes of denitrogenation with $100 \%$ oxygen through a facemask following which $2 \%$ propofol and remifentanil with the Master target controlled infusion was given (Orchestra ${ }^{\circledR}$ Base Primea; Fresenius-MCM GmbH, Germany) after administration of lidocaine (40 mg). After confirming loss of consciousness, rocuronium $(0.5 \mathrm{mg} / \mathrm{kg})$ was administered. Endotracheal intubation was then performed without any complications. After verifying that patient continued to have clear breath sounds in both lung fields, the tube was fixed at a depth of $20 \mathrm{~cm}$ at the level of incisor teeth. The $\mathrm{ETCO}_{2}$ was monitored by capnogram and maintained at 35-40 mmHg.

During the operation, $700 \mathrm{ml}$ of Hartmann solution was administered. After discontinuing the anesthetics, she began to respirate spontaneously. A mixture of glycopyrrolate (0.4 $\mathrm{mg}$ ) and pyridostigmine (15 mg) were administered for muscle recovery and the endotracheal tube was carefully extubated. She was awake fully and had no complaints of any discomfort or respiratory disturbance except for the surgical site pain, and she was therefore transported to the post anesthetic care unit. Oxygen at $5 \mathrm{~L} / \mathrm{min}$ was administered through a Venturi mask when the pulse oximetry indicated a $\mathrm{SpO}_{2}$ of $97 \%$.

Fifteen minutes after extubation, the pulse oximeter indicated $\mathrm{SpO}_{2}$ of $70 \%$. A sudden onset of dyspnea and hemoptysis developed. The patient was promptly intubated and approximately $50 \mathrm{ml}$ of fresh blood was suctioned through the endotracheal tube. A bronchoscopy was immediately performed to determine the cause of hemoptysis and hypoxia. The apparent bleeding was localized to originate from right upper lobe in the beginning, but the bleeding continued to become bilaterally with no identifiable bleeding focus (Fig. 1). The chest radiograph revealed bilateral diffuse alveolar infiltrates (Fig. 2). Arterial blood gas analysis after endotracheal intubation and mechanical ventilator applied were: arterial oxygen tension $\left(\mathrm{PaO}_{2}\right), 55$ mmHg; arterial carbon dioxide tension $\left(\mathrm{PaCO}_{2}\right), 47$ mmHg; $\mathrm{pH}, 7.31 ; \mathrm{Hb}, 10.5 \mathrm{~g} / \mathrm{dl}$.

The patient was transferred to the intensive care unit (ICU) due to her unstable vital signs. The vital signs measured upon arrival showed a blood pressure of $80 / 50 \mathrm{mmHg}$, heart rate of $162 / \mathrm{min}$ and $\mathrm{SpO}_{2}$ of $90 \%$ and Swan-Ganz catheter was inserted. The blood test results revealed the following: $\mathrm{Hb}, 8.7$ g/dl; hematocrit, 26\%; platelet count, 57,000/ $\mathrm{mm}^{3}$; WBC count,
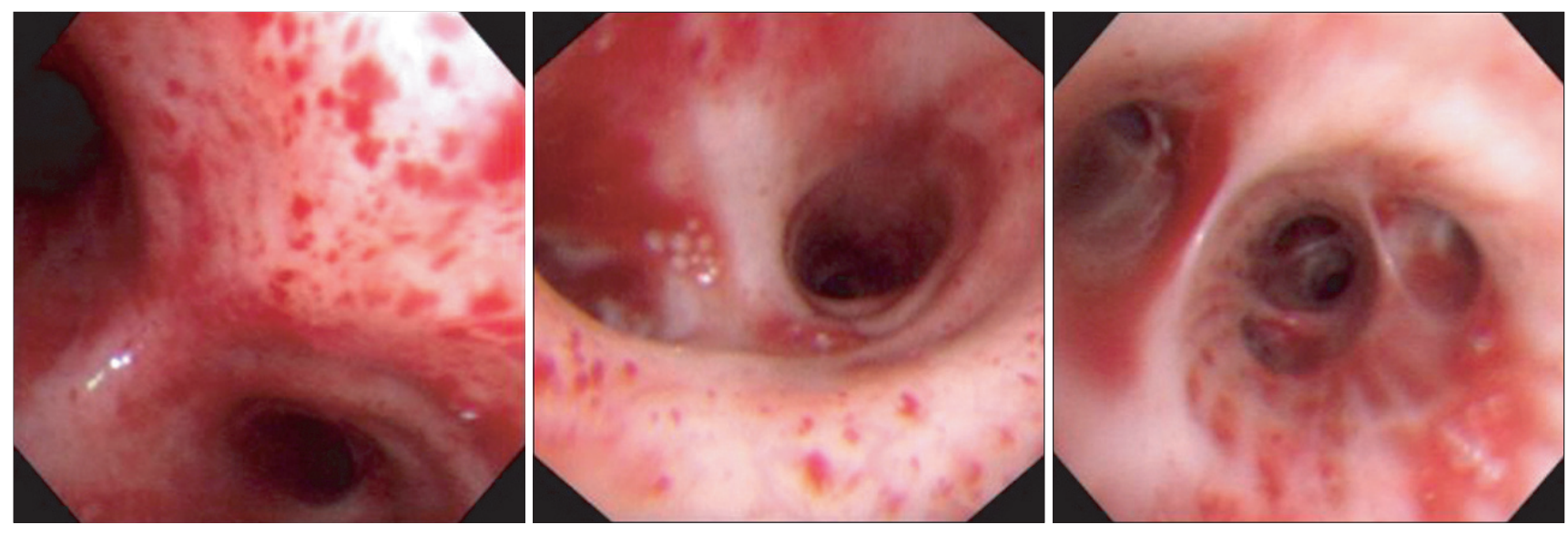

Fig. 1. Fiberoptic bronchoscope showed the generally diffuse fresh blood at bronchial trees. 

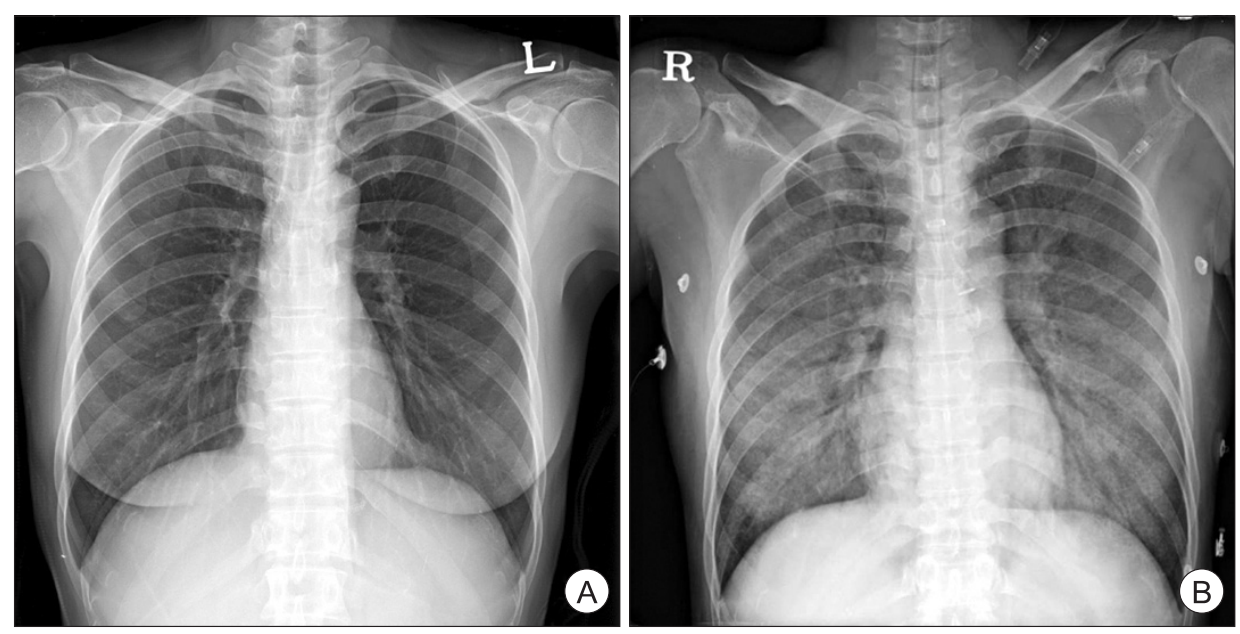

Fig. 2. Preoperative chest PA film appeared within normal limits (A) but postoperative chest AP view demonstrated the bilateral diffuse patchy infiltrates (B).

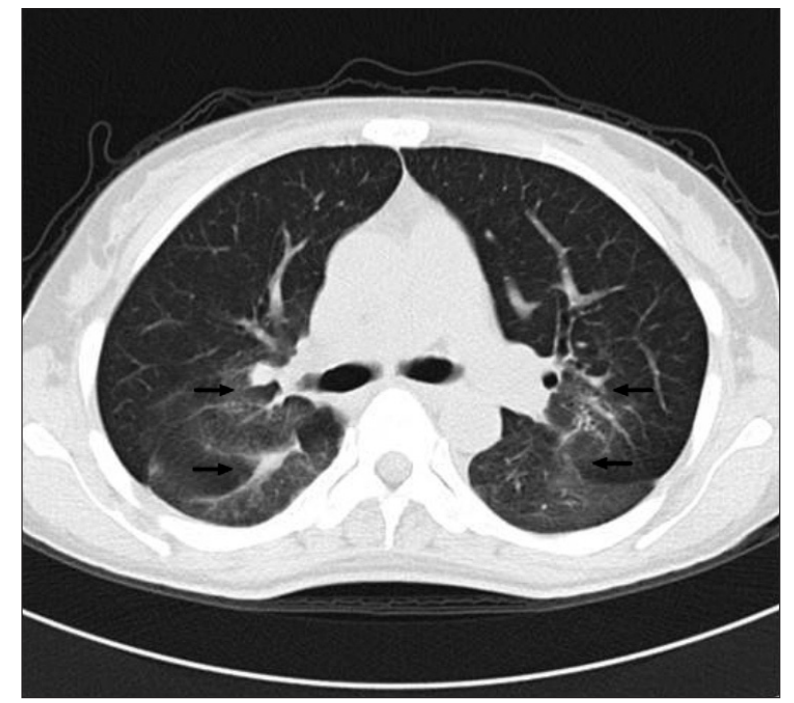

Fig. 3. Chest CT scan taken 1 week after the hemoptysis episode demonstrated multifocal minimal fibrosis and ground glass opacities to be regarded as fibrotic sequel of nonspecific inflammation.

$900 / \mathrm{mm}^{3}$; absolute neutrophil, $640 / \mathrm{mm}^{3}$. The PT was $19.8 \mathrm{sec}$ and the activated PTT was $37.1 \mathrm{sec}$. The vasopressor drugs such as dopamine, norepinephrine and vasopressin were infused to stabilize the blood pressure and hydrocortisone (300 $\mathrm{mg}$ ) was injected. Five units of packed RBCs, 6 units of fresh frozen plasmas and 10 units of platelets were transfused for 7 hours. A bronchoscope was performed again at the ICU by pulmonologist after the vital signs were stable. The bronchoscopy revealed presence of alveolar hemorrhage in both lungs and no focal bleeding sources again. Urinary analysis with microscope and serologic laboratory were checked.

Five days after the intubation, the patient sufficiently recovered and subsequently mechanical ventilation was discontinued. On the postoperative day 6 , there was no evidence of alveolar hemorrhage by bronchoalveolar lavage (BAL). Bilateral infiltration resolved and confirmed by high resolution computed tomography (HRCT) scan images (Fig. 3). The patient became symptom-free and was discharged from the hospital 20 days after the surgery. S-1 was excluded from the chemotherapy regimen in this patient and no recurrences of symptoms were observed after discharge.

\section{Discussion}

The term 'diffuse alveolar hemorrhage' refers to a distinct form of pulmonary hemorrhage that originates from the pulmonary microcirculation including alveolar capillaries, arterioles, and venules [1]. It is also known by names such as intrapulmonary hemorrhage, diffuse pulmonary hemorrhage, pulmonary alveolar hemorrhage, pulmonary capillary hemorrhage, alveolar bleeding, or microvascular pulmonary hemorrhage [2]. Dyspnea, cough, hemoptysis, and new alveolar infiltrates in conjunction with bloody BAL establish the diagnosis of DAH.

DAH can occur in association with various drugs and a wide variety of clinical disorders, many of which have overlapping features of glomerulonephritis, immune complex, and antiglomerular basement membrane disease [1]. Since the serological investigations and renal ultrasound were unremarkable for immune or renal disease in our case, together with the careful exclusion of these causes, several elements led to the suspicion that her lung damage was an expression of drug-induced adverse reaction.

Drug-induced lung injury may represent three mechanisms such as an immune or hypersensitivity reaction, an injury to the alveolar capillary basement membrane, or a coagulation defect [3]. In this case, there was no clinical or laboratory evidence of vasculitis and preoperative thrombocytopenia. We suspect that the drug may have a role to play in causing DAH [3] in this 
patient. The patient's manifestation of DAH in the setting of total gastrectomy and adjuvant chemotherapy raises a question of the possible predisposition of drug toxicity by cytotoxic drugs.

A majority of anti-neoplastic drugs have the potential to induce pulmonary toxicity, involving lung parenchyma, airways, pleura, and pulmonary circulation [4]. S-1 is a recently approved oral anti-neoplastic agent containing prodrug 5 -FU that has been a first line drug in the therapy of gastrointestinal cancers [5]. The major adverse effects of S-1 documented and reported so far are hematological and gastrointestinal nature. There are a few reports of pulmonary complication of S-1 available. S-1 and other cytoxic agents, 5 -FU, have been reported to cause the interstitial lung disease which may be resolved by corticosteroid [6-8]. Thus, increasing the probability of DAH to be caused by $\mathrm{S}-1$, although the exact component of the drug that is implicated was not identified by drug lymphocyte stimulation test (DLST) $[4,9]$. As far as the pathogenesis is concerned, a positive DLST result on 5-FU does not always automatically cause lung injuries. It seems that the direct toxic effect of the drugs on the parenchymal structures is responsible for the lung injuries rather than that of immunologically-driven mechanisms.

For these experiences it becomes extremely important to obtain a full treatment history in all patients who manifest diffuse pulmonary infiltrates. Despite these findings, the timing of the drug administration in relationship to the development of this complication points to its role in the causation of the event. However, it is reported that the pulmonary effects of cytotoxic drugs, such as bleomycin or mitomycin, occurs several months following completion of therapy [10]. The use of oxygen in high concentrations during an unrelated surgery in a patient who was treated previously with bleomycin, and, possibly, other cytotoxic drugs, can result in an acute pulmonary syndrome years after the initial drug exposure $[11,12]$.

Another drug that we could not exclude our suspicion, according to the timing of administration, is propofol, though such toxicity has not been reported previously in the literature. The patient underwent total gastrectomy 3 months ago with same anesthetic method and total infused dose of propofol was twice more than that of this instance. In the endotoxic rat model, pretreatment and simultaneous treatment with propofol provided protection against acute lung injury by inhibiting the TGF-beta1-Smad2 dependent pathway [13].

Transbronchial lung biopsy was intentionally not performed in our patient because of the characteristic finding of DAH (i.e. progressively diffuse hemorrhage) in the beginning. Also, the need for mechanical ventilation and the severity of respiratory failure precluded us from obtaining the biopsy at that point of time. Performing a surgical lung biopsy, although useful in confirming DAH, is generally not suitable to identify the underlying causes [14].
DAH is always a potentially life-threatening situation. Consequently, the first goal in the management of patients with DAH is to achieve or preserve stability of the respiratory status. An urgent bronchoscopy is recommended in patients whose diagnosis is uncertain. A possible management protocol should include supplemental oxygen, bronchodilators, reversal of any coagulopathy, intubation, protective strategies for the less involved lung, and mechanical ventilation. If therapy targets the autoimmune destruction of alveolar capillary membrane and the coexisting autoimmune condition, the application of corticosteroids and immunosuppressive agents should be considered.

This case report describes the unexpected hemoptysis following surgical procedures in patients who underwent recent chemotherapy. This uncommon complication should be considered in the differential diagnosis of unknown etiology of hypoxia during peri-operative periods. The prevalence of this form of pulmonary hemorrhage in patients receiving S-1 chemotherapy is unclear at this time. However, several clinical cases describing such diffuse interstitial pulmonary injury have been reported.

The objective of this write-up is to prime the clinicians on this occurrence and to increase the awareness of the fact that S-1 has the potential to cause lung injury when it is included in chemotherapy. Furthermore, the patients' medication history should be taken into a careful consideration for perioperative evaluation especially in patients with pulmonary complications.

\section{References}

1. Collard HR, Schwarz MI. Diffuse alveolar hemorrhage. Clin Chest Med 2004; 25: 583-92, vii.

2. Fontenot AP, Schwarz MI. Diffuse alveolar hemorrhage. In: Interstitial Lung Disease. 4th ed. Edited by Schwartz MI, King TE Jr: Hamilton, B.C. Decker, Inc. 2003, pp 632-56.

3. Schwarz MI, Fontenot AP. Drug-induced diffuse alveolar hemorrhage syndromes and vasculitis. Clin Chest Med 2004; 25: 133-40.

4. Dimopoulou I, Bamias A, Lyberopoulos P, Dimopoulos MA. Pulmonary toxicity from novel antineoplastic agents. Ann Oncol 2006; 17: 372-9.

5. Takiuchi H, Ajani JA. Uracil-tegafur in gastric carcinoma: a comprehensive review. J Clin Oncol 1998; 16: 2877-85.

6. Tada Y, Takiguchi Y, Fujikawa A, Kitamura A, Kurosu K, Hiroshima K, et al. Pulmonary toxicity by a cytotoxic agent, S-1. Intern Med 2007; 46: 1243-6.

7. Ohara G, Satoh H, Hizawa N. Pulmonary toxicity by an oral antineoplatic agent, S-1. Intern Med 2008; 47: 187.

8. Trisolini R, Lazzari Agli L, Tassinari D, Rondelli D, Cancellieri A, Patelli M, et al. Acute lung injury associated with 5-fluorouracil and oxaliplatinum combined chemotherapy. Eur Respir J 2001; 18: 243-5.

9. Izumikawa K, Nakano K, Kurihara S, Imamura Y, Yamamoto K, Miyazaki T, et al. Diffuse alveolar hemorrhage following itracona- 
zole injection. Intern Med 2010; 49: 497-500.

10. Lesesne JB, Rothschild N, Erickson B, Korec S, Sisk R, Keller J, et al. Cancer-associated hemolytic-uremic syndrome: analysis of 85 cases from a national registry. J Clin Oncol 1989; 7: 781-9.

11. Nalos PC, Kass RM, Gang ES, Fishbein MC, Mandel WJ, Peter T. Life-threatening postoperative pulmonary complications in patients with previous amiodarone pulmonary toxicity undergoing cardiothoracic operations. J Thorac Cardiovasc Surg 1987; 93: 90412.

12. Ingrassia TS 3rd, Ryu JH, Trastek VF, Rosenow EC 3rd. Oxygen- exacerbated bleomycin pulmonary toxicity. Mayo Clin Proc 1991; 66: 173-8.

13. Gao J, Zhao WX, Xue FS, Zhou LJ, Xu SQ, Ding N. Early administration of propofol protects against endotoxin-induced acute lung injury in rats by inhibiting the TGF-beta1-Smad2 dependent pathway. Inflamm Res 2010; 59: 491-500.

14. Travis WD, Colby TV, Lombard C, Carpenter HA. A clinicopathologic study of 34 cases of diffuse pulmonary hemorrhage with lung biopsy confirmation. Am J Surg Pathol 1990; 14: 1112-25. 